\title{
Non-Local Quantum Theory of the Scalar Field
}

\author{
G. V. EFimov \\ Joint Institute for Nuclear Research, Dubna \\ Received November 8, 1966
}

\begin{abstract}
A scheme of the construction of the $S$-matrix according to the perturbation theory which is free of the ultraviolet divergences is suggested by the example of the one-component scalar quantized field. The causality is violated in small space-time region. The effects which are due to the causality violation at large distances are described by very high perturbation orders, and are therefore very small in the framework of the perturbation theory.
\end{abstract}

\section{Introduction. The Macrocausality Condition}

As is well known, the main trouble in the quantum field theory due to the perturbation expansion of the S-matrix in the usual Lagrangian of the system quantized fields is to remove ultraviolet divergences. Many attempts have been made to overcome this trouble by refusing the locality principle, as has been first suggested by VATAGIN [1]. However there are many difficulties in the non-local quantum field theory. It seems to us that at present it is far from being completed ${ }^{1}$.

The present paper is also an attempt to remove the ultraviolet divergences by introducting non-locality into the interaction Lagrangian.

One of the main difficulties in constructing the non-local quantum field theory appears to be the formulation of the macrocausality of the $S$-matrix. Though there are intutive considerations that the acausal signal should damp rapidly with increasing time or distance, the requirement on the $S$-matrix behaviour has not yet been formulated sufficiently clearly, as it has been done in the case of the microcausality [3].

It seems to us that a reasonable macrocausality condition imposed on the $S$-matrix would be the following generalization of the microcausality condition [3]. Let $\varphi(x)$ be a field operator. Then the $S$-matrix should satisfy the following condition ${ }^{2}$

$$
\frac{\delta}{\delta \varphi(x)}\left(\frac{\delta S}{\delta \varphi(y)} S^{-1}\right)=0
$$

1 See a review paper on the non-local quantum field theory [2].

${ }^{2}$ From the drivation of the causality condition in [3] it follows that if $S S^{+} \neq 1$ outside the mass shell in the causality condition there appears $S^{-1}$ rather than $S^{+}$. 
outside the regions $G$ and $G_{l}$ where

$$
\begin{gathered}
G: x_{0} \geqq y_{0},(x-y)^{2}>0 \quad \text { causal region; } \\
G_{l}:-l^{2} \leqq(x-y)^{2} \leqq l^{2} \text { non-causal region . }
\end{gathered}
$$

Here $l$ has the meaning of an "elementary" length, $x^{2}=x_{0}^{2}-\mathrm{x}^{2}$.

An additional requirement should be imposed so that expression (1.1) in the region $G_{l}$ would be proportional to relativistically invariant distributions $A_{l}(x-y)$ which would possess the following property. They must transform any functions $f(x)$ different from zero in some restricted space-time region $G_{f}$ into functions $F(x)=\int d y A_{l}(x-y) f(y)$ different from zero only in a somewhat larger restricted space-time region $G_{F}=G_{f}+\delta G_{f}$. The region $\delta G_{f}$ should be restricted and completely located inside the region $G_{f l}$ such that $x \in G_{f l}$, if $-l^{2} \leqq(x-y)^{2} \leqq l^{2}$ where $y \in G_{f}$. The shape of this "difused" region $G_{F}$ should depend only on the behaviour of the function $f(x)$ in the region $G_{f}$.

Such a definition of macrocausality seems to be rather satisfactory since it is relativistically invariant and free from the usual objections that the dependence of expression (1.1) on the interval $s=(x-y)^{2}$ outside the causal region $G(1.2)$ postulates the existence of non-causal signals whatever large the distances would be.

As will be shown in what follows such distributions exist. However, we have not succeeded in satisfying completely the macrocausality condition formulated. It turned out that in expanding the $S$-matrix in the coupling constant the non-causal region $G_{l}$ enlarges with increasing perturbation order and in the $n$-th order reaches the value $G_{(n-1) l}$. This means that within the complete power series the $S$-matrix does not satisfy, strictly speaking, the causality condition formulated.

However, from the physical point of view the problem may be formulated in such a way that the degree of the causality violation would satisfy the usual requirements imposed on non-local theories. Indeed, using the Lagrangian of the quantized field system, we have to construct the expansion the $S$-matrix in the small coupling constant. Stress that we are interested only in the perturbation series, nothing can be said about the properties of the series as a whole in the framework of the present day methods. Therefore it may be required that causality violations at macroscopic distances described by higher perturbation orders be negligibly small. Usually in the non-local theory one demands that the non-local signals damp sufficiently rapidly as a function of time or distance, e.g. like $e^{-\Lambda t}$ where $\Lambda$ is the cutoff momentum. In the case of weak interactions (where $\Lambda$ is usually about $\sim 100 \mathrm{GeV}$ ) the effect of the non-causal signal is extremely weak, namely $\sim e^{-10^{11}}$, if times are of the order of atomic size, i.e. $t \sim 10^{-18} \mathrm{sec} \sim 1 \mathrm{ev}^{-1}$. Let us look what happens in our scheme. We consider again weak interactions. The quan- 
tity $G l^{-2}$ is a small parameter of the perturbation series, $G$ being the weak interaction constant, $l$ is the elementary length. It is natural to assume that $G l^{-2}<1$. Let e.g. $G l^{-2}$ be equal to $e^{-1}$. The causality violation at distances of the order of the atomic size will be described in the $n$-th perturbation order, where $n \sim \frac{r}{l} \sim 10^{11}$ if as before we choose $r \sim 10^{-8} \mathrm{~cm} \sim 1 \mathrm{ev}^{-1}$ and $l \sim 10^{-2} \cdot \mathrm{Gev}^{-1}$. This means that the magnitude of the non-local effect at distances of the order of atomic size is about $\sim\left(G l^{2}\right)^{n} \sim e^{-10^{11}}$. In other words, the effect is as small as in the usual non-local theories which are worth studying.

Thus, from the physical point of view the suggested formulation of a non-local theory for interactions with small coupling constant is, in our opinion, admissible. In the present paper we consider an one-component scalar field, for simplicity. The generalization of the suggested procedure to the fermion field is not difficult. In $\S 2$ problem is formulated. In $\S 3$ a class of non-local operators is chosen and in $\S 4$ the properties of the classes of test functions and distributions are studied. $\S 5$ is devoted to the construction of the perturbation series for the $S$-matrix. It is shown in $\S 6$ that the $S$-matrix is unitary and in $\S 7$ that it satisfies the causality condition formulated.

\section{Formulation of the Problem}

We consider an one-component scalar field $\varphi(x)$ which is described by the interaction Lagrangian of the form:

$$
\mathscr{L}(x)=\mathscr{L}_{0}(x)+\mathscr{L}_{I}(x) .
$$

Here $\mathscr{L}_{0}(x)$ is a usual free-field Lagrangian, $\mathscr{L}_{I}(x)$ is the interaction Lagrangian of the field $\varphi(x)$. In the case of the usual local field theory the interaction Lagrangian is a polynomial of the field $\varphi(x)$, e.g. $\mathscr{L}_{I}(x)$ $=g \varphi^{4}(x)$.

Consider the following problem. In the interaction Lagrangian we replace the field $\varphi(x)$ by the field $\Phi(x)$ defined as follows:

$$
\begin{gathered}
\Phi(x)=\int d y A(x-y) \varphi(y)=A\left(\square_{x}\right) \varphi(x), \\
A(x-y)=A\left(\square_{x}\right) \delta^{(4)}(x-y),
\end{gathered}
$$

where $A\left(\square_{x}\right)$ is an operator of $\square_{x}=\frac{\partial^{2}}{\partial x_{0}^{2}}-\frac{\partial^{2}}{\partial x_{1}^{2}}-\frac{\partial^{2}}{\partial x_{2}^{2}}-\frac{\partial^{2}}{\partial x_{3}^{2}}$.

We make the following transformations. The $S$-matrix can be formally written in the form of the $T$-product:

$$
S=T \exp \left\{-i \int d x \mathscr{L}_{I}(x)\right\}
$$

where $\mathscr{L}_{I}(x)$ is now the polynomial of the field $\Phi(x)$, e.g. $\mathscr{L}_{I}(x)=g \Phi^{4}(x)$. 
We expand the $S$-matrix in a power series in the coupling constant and, according to the Wick theorem, we pass to the $N$-product of the field operators $\Phi(x)$ where the chronological pairing of the operators $\Phi(x)$ implies the so-called "Wick- $T$-product"

$$
\begin{aligned}
& D_{c}(x-y)=\overparen{\Phi(x) \Phi}(y)=A\left(\square_{x}\right) A\left(\square_{y}\right) \overparen{\varphi(x) \varphi(y)} \\
& \quad=A\left(\square_{x}\right) A\left(\square_{y}\right) \Delta_{c}(x-y)=\frac{1}{(2 \pi)^{4} i} \int \frac{d^{4} p\left[A\left(-p^{2}\right)\right]^{2}}{p^{2}-m^{2}+i \varepsilon} e^{i p(x-y)}
\end{aligned}
$$

Thus we obtain the ordinary perturbation series with the only difference that the usual causal functions of the scalar field are replaced by the function (2.5).

Our problem in the following. Is it possible to find the operators $A(\square)$ so that the functions $\left[A\left(-p^{2}\right)\right]^{2}$ play the role of the cutoff function in the perturbation series, i.e. the integrals corresponding to any Feynman graphs converge and the $S$-matrix obeys unitarity and causality conditions?

\section{Properties of the Operators $\boldsymbol{A}(\square)$}

Let us study the properties of the operators $A(\square)$. We assume that the operators $A(\square)$ can be represented as an infinite series in powers $\square$ :

$$
A(\square)=\sum_{n=0}^{\infty} \frac{a_{n}}{(2 n) !} \square^{n}
$$

Then the Fourier transform of this operator is written as:

$$
A(z)=\sum_{n=0}^{\infty} \frac{a_{n}}{(2 n) !} z^{n} ; \quad z=-p^{2} .
$$

What requirements the function $A(z)$ should obey? First of all note that $A(z)$ treated as a function of the complex variable $z=-p^{2}$ must be an entire function. Otherwise any singularities of the function $A(z)$ for finite $z$ will lead to the appearance of some additional non-physical singularities in the physical process amplitudes. This will mean that the $S$-matrix is not unitary.

Thus, the function in eq. (3.2) is an entire function. We distinguish three cases:

$$
\begin{aligned}
& \varlimsup_{n \rightarrow \infty}\left|a_{n}\right|^{\frac{1}{n}}=0, \\
& \varlimsup_{n \rightarrow \infty}\left|a_{n}\right|^{\frac{1}{n}}=\text { const }<\infty, \\
& \varlimsup_{n \rightarrow \infty}\left|a_{n}\right|^{\frac{1}{n}}=\infty .
\end{aligned}
$$


In case I the functions $A(z)$ are entire functions of the order $\gamma<\frac{1}{2}$, i.e.

$$
|A(z)|<e^{a|z|^{\gamma}}, \quad \gamma<\frac{1}{2}
$$

where $a$ is a positive number. The example of such functions is the function

$$
E_{\alpha}(z)=\sum_{n=0}^{\infty} \frac{z^{n}}{\Gamma(\alpha n+1)}, \quad \alpha>2 .
$$

According to the theory of entire functions (see e.g. [4]) it is known that for these functions there is not any direction in the complex plane $z$, along which they could decrease. Consequently, they cannot play the role of the cutoff functions and in this case it is impossible to make the perturbation theory finite.

On the other hand, it can be shown by extending the results of the Matmand [5] paper to a relativistic case that the operators $A(\square)$ with the coefficients $a_{n}$ obeying condition (3.3.I) are local. Thus, this means that it is impossible to construct a perturbation theory without ultraviolet divergences, introducing into the interaction Lagrangian instead of $\varphi(x)$ the local field $\Phi(x)$.

In case II the functions $A(z)$ are entire functions of the order $\frac{1}{2}$, i.e.

$$
|A(z)| \leqq e^{a \sqrt{|z|}}
$$

Such functions may have only one direction in the complex plane along which they may decrease. This is just the case which will be discussed below.

In case (III) the functions $A(z)$ are entire ones the order of which is higher $\operatorname{than} \frac{1}{2}$, i.e.

$$
|A(z)| \leqq e^{h(|z|)}
$$

where $h(|z|)$ is a positive function satisfying the condition $h(|z|)>$ $>a|z|^{\frac{1}{2}}$ at $|z| \rightarrow \infty$ for any $a>0$. Such functions may have some regions where they decrease when $|z| \rightarrow \infty$ so that they may be chosen as the cutoff functions for the construction of a finite unitary perturbation theory. However it may be shown that the field operators $\Phi(x)$ are no longer local, i.e. the behaviour of the operator of the field $\Phi(x)$ at the point $x=0$ is determined by the behaviour of the field operator $\varphi(x)$ over the whole $x$-space. In other words, the differential operator $A(\square)$ in (3.3.III) is, in fact, an integral operator whose kernel differs from zero over the whole $x$-space. Therefore this theory would not satisfy the causality condition. 


\section{The Class of Test Functions and the Distributions}

We consider the distributions

$$
A_{\lambda}(x-y)=A_{\lambda}\left(\square_{x}\right) \delta^{(4)}(x-y)
$$

for which the operator $A_{\lambda}(\square)$ of the type (3.III) can be written in the form

$$
\begin{gathered}
A_{\lambda}(\square)=\int_{\varrho^{2}<\lambda^{2}} d^{4} \varrho a\left(\varrho^{2}\right) \exp \left(i \varrho_{0} \frac{\partial}{\partial x_{0}}+\varrho \frac{\partial}{\partial \mathbf{x}}\right) \\
=(2 \pi)^{2} \int_{0}^{\lambda} d \beta \beta^{2} a\left(\beta^{2}\right) \frac{J_{1}(\beta \sqrt{\square})}{\sqrt{\square}} \\
\text { or } \begin{aligned}
A_{\lambda}(\square) & =\int_{\varrho^{2}<\lambda^{2}} d^{4} \varrho \\
& =(2 \pi)^{2} \int_{0}^{\lambda} d \beta \varrho^{2} a\left(\beta^{2}\right) \frac{J_{1}(\beta \sqrt{-\square})}{\sqrt{-\square}} .
\end{aligned}
\end{gathered}
$$

Here $\varrho$ is an Euclidean four-vector $\varrho^{2}=\varrho_{0}^{2}+\varrho_{1}^{2}+\varrho_{2}^{2}+\varrho_{3}^{2}, a\left(\varrho^{2}\right)$ is an integrable function of $\varrho^{2}, J_{1}(z)$ is the Bessel function, $\lambda$ is a parameter having the meaning of the "elementary" length, as will be seen below.

In what follows we say that the operators $A_{\lambda}(\square)$ belong to the type (A) or (B) if they are represented in the form (4.2a) or (4.2 b) respectively.

We write the operators $(4.2)$ in the momentum representation

$$
\begin{array}{cc}
A_{\lambda}\left(-p^{2}\right)=(2 \pi)^{2} \int_{0}^{\lambda} d \beta \beta^{2} a\left(\beta^{2}\right) \frac{J_{1}\left(\beta \sqrt{-p^{2}}\right)}{\sqrt{-p^{2}}}, & (\text { type A), } \\
A_{\lambda}\left(-p^{2}\right)=(2 \pi)^{2} \int_{0}^{\lambda} d \beta \beta^{2} a\left(\beta^{2}\right) \frac{J_{1}\left(\beta \sqrt{p^{2}}\right)}{\sqrt{p^{2}}}, & (\text { type B). }
\end{array}
$$

We note that if the operator $A_{\lambda}(\square)$ belongs to type (A) then the function $A_{\lambda}\left(-p^{2}\right)$ decreases at $p^{2} \rightarrow-\infty$ and increases at $p^{2} \rightarrow+\infty$. If the operator $A_{\lambda}(\square)$ belongs to type (B) the function $A_{\lambda}\left(-p^{2}\right)$ decreases at $p^{2} \rightarrow+\infty$ and increases at $p^{2} \rightarrow-\infty$.

Now let us investigate the properties of the class of test functions. This class is denoted by $D$ and the class of distributions by $D^{\prime}$. First of all we require that the test functions $f(x) \equiv f\left(x_{0}, \mathbf{x}\right) \equiv f\left(x_{0}, x_{1}, x_{2}, x_{3}\right)$ be reals and decrease at infinity. The following functional must be determined unambiguously in the class of test functions

$$
\begin{gathered}
\left(A_{\lambda}, f\right)=\int d y A_{\lambda}(x-y) f(y)=A_{\lambda}\left(\square_{x}\right) f(x)= \\
=\int_{\varrho^{2}<\lambda^{2}} d^{4} \varrho a\left(\varrho^{2}\right) \exp \left(i \varrho_{0} \frac{\partial}{\partial x_{0}}+\varrho \frac{\partial}{\partial \mathbf{x}}\right) f(x) \\
=\int_{\varrho^{2}<\lambda^{2}} d^{4} \varrho a\left(\varrho^{2}\right) f\left(x_{0}+i \varrho_{0}, \mathbf{x}+\varrho\right) \\
\left(A_{\lambda}, f\right)=\int_{\varrho^{2}<\lambda^{2}} d^{4} \varrho a\left(\varrho^{2}\right) f\left(x_{0}+\varrho_{0}, \mathbf{x}+i \varrho\right), \quad(\text { type B) } .
\end{gathered}
$$


We require that the functionals $\left(A_{\lambda}, f\right)$ in $(4.4 \mathrm{a})$ and $(4.4 \mathrm{~b})$ should be unambiguously determined in any Lorentz frame of reference and for any value of the parameter $\lambda$. Then the class of test functions must consist of entire analytical functions $f\left(z_{0}, z_{1}, z_{2}, z_{3}\right)$ with respect to each argument $z_{0}, z_{1}, z_{2}, z_{3}$.

Thus we shall assume that the class of test functions consists of the entire functions $f(z)$ which decrease along any direction in the $z$-plane outside the region $|\operatorname{Re} z|<d$ where $d$ is a certain number, i.e.

$$
\lim _{z \rightarrow \infty} z f(z)=0 \quad \text { when } \quad|\operatorname{Re} z|<d .
$$

We give the example of such a function

$$
f(z)=\frac{1}{z} \int_{0}^{\infty} \frac{d u \operatorname{Sin} u z}{\Gamma(\varrho u+1)} .
$$

Here $f(z)=O\left(\frac{1}{z^{2}}\right)$ in the range $|\operatorname{Re} z|>\frac{\pi}{\varrho}$ at $z \rightarrow \infty$. We give a final definition of the class $(D)$ of test functions:

1. Each $f(x) \in D$ is an entire analytical function with respect to each its argument $x_{j}$ and satisfies the condition (4.5) where $d$ depends generally speaking on the function $f(x)$.

2. Each $f(x) \in D$ is real on the real axis.

3. The sequence of the functions $f_{n}(x) \in D$ converges to zero in a certain region $G$ if all functions of the sequence tend uniformly to zero in this region.

For a further consideration we have to know the local properties of the distributions $A_{\lambda}(x-y)$. To determine the local properties of the distributions it is necessary to have the test functions localized in a certain bounded region of the $x$-space or simply at any point. Such functions are absent in the class $D$. However, we can choose the sequence $\left\{f_{n}(x, y)\right\}$ so that each $f_{n}(x, y)$ belong to $D$ but the limiting function

$$
f(x, y)=\lim _{n \rightarrow \infty} f_{n}(x, y)
$$

no longer belongs to the class $D$ and vanishes at all points $x \neq y$. If, in addition, the functions $f_{n}(x, y)$ are normalized to the condition

$$
\int d^{4} x f_{n}(x, y)=1
$$

then the sequence introduced gives the representation of the $\delta$-function in $D$. As an example of such a sequence we choose

$$
f_{v}(x, y)=f_{v}(x-y)=\prod_{j=0}^{3} \frac{1}{v_{j}} f_{j}\left(\frac{x_{j}-y_{j}}{v_{j}}\right)
$$

where $v$ implies four independent positive parameters $v_{0}, v_{1}, v_{2}, v_{3}$ with 
respect to each $x_{j}$ and $f_{j} \in D$ and satisfies the condition (4.7). Then

$$
\lim _{\nu \rightarrow 0} f_{\nu}(x-y)=\delta(x-y) \text {. }
$$

Let us consider the sequence

$$
g_{v}(x-y)=\int d^{4} x^{\prime} A_{\lambda}\left(x-x^{\prime}\right) f_{v}\left(x^{\prime}-y\right)=A_{\lambda}\left(\square_{x}\right) f_{v}(x-y)
$$

where the function $f_{v}(x-y)$ in chosen according to (4.8). Let us look where the function $g(x-y)=\lim _{v \rightarrow 0} g_{v}(x-y)$ vanishes if $\lim _{\nu \rightarrow 0} f_{v}(x-y)=0$ for all $x \neq y$.

First of all we investigate the properties of the operators $A_{\lambda}(\square)$ of the type (A). For simplicity we put $y=0$. We get

$$
g(x)=\lim _{\nu \rightarrow 0} g_{v}(x)=\lim _{\nu \rightarrow 0} \int_{\varrho^{2}<\lambda^{2}} d^{4} \varrho a\left(\varrho^{2}\right) f_{v}\left(x_{0}+i \varrho_{0}, \mathbf{x}+\varrho\right) .
$$

Here we can pass to the limit $v_{1}=v_{2}=v_{3}=0$. According to (4.9) we obtain

$$
g(x)=\lim _{v_{0} \rightarrow 0} \theta\left(\lambda^{2}-\mathbf{x}^{2}\right) \int_{-\sqrt{\lambda^{2}-\mathbf{x}^{2}}}^{\sqrt{\lambda^{2}-\mathbf{x}^{2}}} d \varrho_{0} a\left(\mathbf{x}^{2}+\varrho_{0}^{2}\right) \frac{1}{v_{2}} f\left(\frac{x_{0}+i \varrho_{0}}{v_{0}}\right) .
$$

According to the property (4.5) of the test functions

Finally

$$
\lim _{v_{0} \rightarrow 0} \frac{1}{v_{0}} f\left(\frac{x_{0}+i \varrho_{0}}{v_{0}}\right)=0 \text { for all } x_{0} \neq 0 \text {. }
$$

$$
g(x)=\lim _{\nu \rightarrow 0} g_{\nu}(x)=0
$$

outside the region $x_{0}=0, \mathbf{x}^{2}<\lambda^{2}$. Thus when the operator $A_{\lambda}(\square)$ of the type (A) acts on the sequence $\left\{f_{v}(x-y)\right\}$ the limiting function of which vanishes everywhere, the exept the point $x=y$, we get another sequence $\left\{g_{v}(x-y)\right\}$ the limiting function of which vanishes outside a certain bounded space-like region $G_{A}\left(x_{0}=y_{0}, \mathbf{x}=\mathbf{y}+\boldsymbol{\varrho}\right.$, where $\left.\boldsymbol{\varrho}^{2}<\lambda^{2}\right)$ around the point $y$. The four-volume of this region is zero.

Now let us consider the sequence (4.10) in the case when the operator $A_{\lambda}(\square)$ belongs to the type (B). We have

$$
g(x)=\lim _{\nu \rightarrow 0} \int_{\varrho^{2}<\lambda^{2}} d^{4} \varrho a\left(\varrho^{2}\right) f_{\nu}\left(x_{0}+\varrho_{0}, \mathbf{x}+i \boldsymbol{\varrho}\right) .
$$

Here we can pass to the limit $v_{0}=0$. According to (4.9) we get

$$
g(x)=\lim _{v_{1}, \nu_{2}, \nu_{3} \rightarrow 0} \theta\left(\lambda^{2}-x_{0}^{2}\right) \int_{\varrho^{2}<\lambda^{2}-x_{0}^{2}} d \underline{\varrho} a\left(x_{0}^{2}+\varrho^{2}\right) \prod_{j=1}^{3} \frac{1}{v_{j}} f_{j}\left(\frac{x_{j}+i \varrho_{j}}{v_{j}}\right) .
$$

Owing to the property (4.5)

$$
\lim _{v_{1}, v_{2}, v_{3} \rightarrow 0} \prod_{j=1}^{3} \frac{1}{v_{j}} f_{j}\left(\frac{x_{j}+i \varrho_{j}}{v_{j}}\right)=0 \text { for all } \mathbf{x} \neq 0 .
$$

4 Commun. math. Phys., Vol. 5 
Finally

$$
g(x)=\lim _{\nu \rightarrow 0} g_{\nu}(x)=0
$$

outside the region $x_{0}^{2}<\lambda^{2}, \mathrm{x}=0$. The action of the operator of the type (B) on the sequence $\left\{f_{v}(x-y)\right\}$ leads to a sequence $\left\{g_{v}(x-y)\right\}$ the limiting function of which is zero outside the bounded time-like region $G_{B}$ $\left(x_{0}=y_{0}+\tau\right.$, where $\left.\tau^{2}<\lambda^{2}, \mathbf{x}=\mathbf{y}\right)$ around the point $y$. The four-volume of this region is zero too.

Stress that there exist various sequences $\left\{f_{v}(x-y)\right\}$ which satisfy the condition (4.6). Such are all sequences which can be obtained from (4.8) by the Lorentz transformations. Under the action of the operator $A_{\lambda}(\square)$ each such sequence will transform into a new sequence. The latter reduces to the function equal to zero outside a bounded region connected by the appropriate Lorentz transformation with the region for the sequence (4.8). All these bounded regions obtained from sequences $\left\{f_{v}(x-y)\right\}$ lie inside the hyperboloid:

$$
-\lambda^{2} \leqq(x-y)^{2} \leqq \lambda^{2}
$$

Thus the physical meaning of the distributions $A_{\lambda}(x-y)$ is the following. Let $\varphi(x)$ be a certain field appears and then disappears at time $y_{0}$ in the space point $\mathbf{y}$. Then, due to the "propagator" $A_{\lambda}(x-y)$ this field will affect a certain bounded region with zero four-volume which lies completely inside the hyperboloid (4.17). The shape of this region will depend on the "micro-shape" of the impulse of the field $\varphi(x)$.

In conclusion we describe one more important property of the distributions (4.1) which the tempered functions do not possess. It is possible to define unambiguously the product of the distributions (4.1) with the operator $A_{\lambda}(\square)$ of the form $(4.2 \mathrm{a})$ or $(4.2 \mathrm{~b})$ :

$$
C_{\lambda}(x-y)=-i A_{\lambda}^{(1)}(x-y) A_{\lambda}^{(2)}(x-y)
$$

where the indices 1 and 2 denote the functions $a_{1}\left(\varrho^{2}\right)$ and $a_{2}\left(\varrho^{2}\right)$ in the integrand of (4.2).

We construct the distributions by an improper transition to the limit. Approximate these distributions by the following regular functions:

$$
\begin{gathered}
A_{\lambda}^{\varepsilon}(x-y)=\frac{1}{(2 \pi)^{4}} \int d^{4} p e^{i p x} A_{\lambda}\left(-p^{2}\right) R^{\varepsilon}\left(p^{2}\right), \\
R^{\varepsilon}(\xi)=\exp \left\{-\varepsilon(\xi+i)^{\frac{1}{2}+v} e^{-i \pi \sigma}\right\}
\end{gathered}
$$

where $0<v<\sigma<\frac{1}{2}$. For the regularizing function $R^{\varepsilon}(\xi)$ at large $\xi$ the estimate

$$
\left|R^{\varepsilon}(\xi)\right| \sim \exp \left\{-\varepsilon|\xi|^{\frac{1}{2}+v} \cos \left(\pi \sigma-\left(v+\frac{1}{2}\right) \arg \xi\right)\right\}
$$


is valid. In other words, $R^{\varepsilon}(\xi)$ is analytical and falls out faster than the linear exponential in the upper half-plane of the complex variable $\xi$. The integral (4.19) well converges at $\varepsilon>0$ and determine a certain function $A_{\lambda}^{\varepsilon}(x-y)$.

Now let us consider the product

$$
C_{\lambda}^{\varepsilon}(x-y)=-i A_{\lambda}^{(1) \varepsilon}(x-y) A_{\lambda}^{(2) \varepsilon}(x-y)
$$

and show that at $\varepsilon \rightarrow 0$ there exists a limit for the functional $\left(C_{\lambda}^{\varepsilon}, f\right)$ where $f$ belongs to the class $D$. We perform some simple transformations

$$
\begin{aligned}
\left(C_{\lambda}^{\varepsilon}, f\right) & =\int d^{4} y C_{\lambda}^{\varepsilon}(x-y) f(y) \\
& =-i \int d^{4} y A_{\lambda}^{(1) \varepsilon}(x-y) A_{\lambda}^{(2) \varepsilon}(x-y) f(y) \\
& =-i \int d^{4} p e^{i p x} \tilde{f}(p) \int d^{4} q A_{\lambda}^{(1) \varepsilon}\left(-q^{2}\right) A_{\lambda}^{(2) \varepsilon}\left(-(p-q)^{2}\right)
\end{aligned}
$$

where

$$
A_{\lambda}^{\varepsilon}\left(-q^{2}\right)=A_{\lambda}\left(-q^{2}\right) R^{\varepsilon}\left(q^{2}\right) .
$$

Since the function $f(y) \in D$ then its Fourier transform $f$ decreases along the real axis $p$ more rapidly than the linear exponential. In the last integral over $d^{4} q$ one can pass to the Euclidean metric by turning the contour of integration over $q_{0}$ at the angle $\frac{\pi}{2}$ or over $q_{j}(j=1,2,3)$ at $-\frac{\pi}{2}$ depending on which type (A) or (B) the operators $A_{\lambda}^{(1)}(\square)$ and $A_{\lambda}^{(2)}(\square)$ belong to. The possibility of turning the contour is proved by the property (4.21) of the regularizing function $R^{\varepsilon}$. For definiteness we assume that $A_{\lambda}^{(1)}$ and $A_{\lambda}^{(2)}$ belong to the type (A). Then after turning the contour of integration over $q_{0}$ at $\frac{\pi}{2}$ we get

$$
\begin{aligned}
& \left(C_{\lambda}^{\varepsilon}, f\right)=\int d^{4} p e^{i p x} f(p) C_{\lambda}^{\varepsilon}\left(p^{2}\right), \\
& C_{\lambda}^{\varepsilon}\left(p^{2}\right)=\int d^{4} q_{E} A_{\lambda}^{(1) \varepsilon}\left(+q_{E}^{2}\right) A_{\lambda}^{(2) \varepsilon}\left(+\left(q_{E}-i p\right)^{2}\right),
\end{aligned}
$$

where $q_{E}$ is the Euclidean vector $q_{E}^{2}=q_{0}^{2}+q_{1}^{2}+q_{2}^{2}+q_{3}^{2}$. Inserting into (4.25) the function $A_{\lambda}^{\varepsilon}\left(+q_{E}^{2}\right)$ according to $(4.2 \mathrm{a})$ we get:

$$
\begin{aligned}
C_{\lambda}^{\varepsilon}\left(-p^{2}\right)= & \int d^{4} q_{E} \int_{\varrho_{1}^{2}<\lambda^{2}} d^{4} \varrho_{1} a_{1}\left(\varrho_{1}^{2}\right) e^{i q_{E} \varrho_{1}} \int_{\varrho_{2}^{2}<\lambda^{2}} d^{4} e_{2} a_{2}\left(\varrho_{2}^{2}\right) e^{i\left(q_{E}-i p\right) \varrho_{2}} \times \\
& \times R^{\varepsilon}\left(-q_{E}^{2}\right) R^{\varepsilon}\left(-\left(q_{E}-i p\right)^{2}\right) .
\end{aligned}
$$

It is seen that here one can go over to the limit $\varepsilon \rightarrow 0$ since the integrand in (4.25) is integrable and the integral (4.24) will be convergent because $f(p)$ rather decreases. We have

$$
\begin{aligned}
& C_{\lambda}\left(-p^{2}\right)=\lim _{\varepsilon \rightarrow 0} C_{\lambda}^{\varepsilon}\left(-p^{2}\right)=\int d^{4} q_{E} \int d_{\varrho_{1}^{2}<\lambda^{2}} d^{4} \varrho_{1} a_{1}\left(\varrho_{1}^{2}\right) e^{i q_{E} \varrho_{1}} \int_{\varrho_{2}^{2}<\lambda^{2}} d^{4} \varrho_{2} a_{2}\left(\varrho_{2}^{2}\right) e^{i\left(q_{E}-i p\right) \varrho_{2}} \\
& =(2 \pi)^{4} \int_{\varrho^{2}<\lambda^{2}} d^{4} \varrho a_{1}\left(\varrho^{2}\right) a_{2}\left(\varrho^{2}\right) e^{p_{0} \varrho_{0}+i p \varrho} .
\end{aligned}
$$

Finally

$$
\left(C_{\lambda}, f\right)=\lim _{\varepsilon \rightarrow 0}\left(C_{\lambda}^{\varepsilon}, f\right)=\int d^{4} y C_{\lambda}(x-y) f(y)
$$


where

$$
\begin{gathered}
C_{\lambda}(x-y)=C_{\lambda}(\square) \delta(x-y), \\
C_{\lambda}(\square)=\int_{\varrho^{2}<\lambda^{2}} d^{4} \varrho c\left(\varrho^{2}\right) \exp \left\{i \varrho_{0} \frac{\partial}{\partial x_{0}}+\varrho \frac{\partial}{\partial \mathbf{x}}\right\}, \\
c\left(\varrho^{2}\right)=a_{1}\left(\varrho^{2}\right) a_{2}\left(\varrho^{2}\right) .
\end{gathered}
$$

Similar formulas are obtained if both distributions $A_{\lambda}^{(1)}$ and $A_{\lambda}^{(2)}$ belong to the type (B). If $A_{\lambda}^{(1)}$ and $A_{\lambda}^{(2)}$ belong to different types then their product can no longer be defined as far as no turning of the contour in the integral (4.25) can let the integrand decrease in the limit $\varepsilon \rightarrow 0$.

\section{The Perturbation Series for the S-Matrix}

As was already mentioned in Section 2 the perturbation series for the $S$-matrix (2.4) is constructed by mean of the usual Feynman graphs, using the function (2.5) instead of the ordinary causal function. In the $x$-space the matrix element of a process in the $n$-approximation of perturbation theory is a sum of the expressions of the type:

$$
F\left(x_{1}, \ldots, x_{n}\right)=\prod_{i, j} D_{c}\left(x_{i}-x_{j}\right)
$$

where $i$ and $j$ are integer from 1 to $n$ according to a suitable choice of the Feynmann diagram. The amplitude $F\left(x_{1}, \ldots, x_{n}\right)$ in $(5.1)$ is a distribution of the class $D^{\prime}$ since

$$
\begin{gathered}
D_{c}(x)=\Delta_{c}(x)+K_{l}(x) \\
K_{l}(x)=\frac{1}{(2 \pi)^{4} i} \int \frac{d^{4} p e^{i p x}}{p^{2}-m^{2}+i \varepsilon}\left\{\left[A_{\lambda}\left(-p^{2}\right)\right]^{2}-1\right\} \\
=\frac{i}{(2 \pi)^{4}} \int d^{4} p K_{l}\left(-p^{2}\right) e^{i p x}=i K_{l}(\square) \delta(x)
\end{gathered}
$$

where $l=2 \lambda$ and the operator $K_{l}(\square)$ belongs to the same type as the operator $A_{\lambda}(\square)$. We assume that the operator $A_{\lambda}(\square)$ is normalized as follows:

$$
A_{\lambda}\left(-m^{2}\right)=1
$$

where $m$ is the mass of the scalar particles considered. The amplitude $F\left(x_{1}, \ldots, x_{n}\right)$ is integrable in the class $D$ test functions.

It is convenient to construct the Fourier transform of the amplitude $F\left(x_{1}, \ldots, x_{n}\right)$ in $(5.1)$ using an improper transition to the limit. Instead of the causal function $D_{c}(x)$ we introduce the regularized function:

$$
\operatorname{reg} D_{c}\left(x_{1}-x_{2}\right)=\frac{1}{(2 \pi)^{4}} \int \frac{d^{4} p e^{i p\left(x_{1}-x_{2}\right)}}{p^{2}-m^{2}+i \varepsilon}\left[A_{\lambda}\left(-p^{2}\right)\right]^{2} R^{\varepsilon}\left(p^{2}\right)
$$

where $R^{\varepsilon}\left(p^{2}\right)$ is chosen as in (4.20). It is easy to show that

where $f(x) \in D$.

$$
\lim _{\varepsilon \rightarrow 0} \int d^{4} x \operatorname{reg} D_{c}(x) f(x)=\int d x D_{c}(x) f(x)
$$


Thus, instead of $F\left(x_{1}, \ldots, x_{n}\right)$ in (5.1) we consider the regularized expression: $\quad F^{\varepsilon}\left(x_{1}, \ldots, x_{n}\right)=\prod_{i, j} \operatorname{reg} D_{c}\left(x_{i}-x_{j}\right)$.

We write the Fourier transform of this function

$\tilde{F}^{\varepsilon}\left(p_{1}, \ldots, p_{n}\right)=\int d^{4} x_{1} \ldots \int d^{4} x_{n} e^{i\left(p_{1} x_{1}+\ldots+p_{n} x_{n}\right)} F^{\varepsilon}\left(x_{1}, \ldots, x_{n}\right)$.

Going over to the momentum representation we get an integral:

$$
\begin{aligned}
\tilde{F}^{\varepsilon}\left(p_{1}, \ldots, p_{n}\right) & =(2 \pi)^{4} \delta\left(p_{1}+\cdots+p_{n}\right) T^{\varepsilon}\left(p_{1}, \ldots, p_{n}\right), \\
T^{\varepsilon}\left(p_{1}, \ldots, p_{n}\right) & =\int \ldots \int \prod_{i} d^{4} l_{i} \prod_{j} \frac{\left[A_{\lambda}\left(-k_{j}^{2}\right)\right]^{2}}{k_{j}^{2}-m^{2}+i \varepsilon} R^{\varepsilon}\left(k_{j}^{2}\right) .
\end{aligned}
$$

$k_{j}$ is the four-momentum corresponding to the given line in the diagram, $l_{i}$ are the four-momenta over which the integration is performed. The integral (5.10) converges for $\varepsilon>0$.

The analytical properties of $R^{\varepsilon}\left(k^{2}\right)$ are such that in (5.16) at $\varepsilon>0$ the contour of integration over the time components $\left(l_{j}\right)_{0}$ can be turned at the angle $\frac{\pi}{2}$ or over space components $\left(l_{j}\right)_{1},\left(l_{j}\right)_{2},\left(l_{j}\right)_{3}$ at the angle $-\frac{\pi}{2}$. The type of the function $A_{\lambda}\left(-k^{2}\right)$ defines which turning is to be made so that to get the finite limit at $\varepsilon \rightarrow 0$. If $A_{\lambda}\left(-k^{2}\right)$ belongs to the type (A), i.e. $A_{\lambda}\left(-k^{2}\right) \rightarrow 0$ at $k^{2} \rightarrow-\infty$, then it is necessary to make the turning over the $\left(l_{j}\right)_{0}$, and in the case $(\mathrm{B})$, i.e. when $A_{\lambda}\left(-k^{2}\right) \rightarrow 0$ at $k^{2} \rightarrow+\infty$, over $\left(l_{j}\right)_{1},\left(l_{j}\right)_{2},\left(l_{j}\right)_{3}$.

Let $A_{\lambda}\left(-k^{2}\right)$ belongs to the type (A). Then in (5.10) one can turn the contour over $\left(l_{j}\right)_{0}$ and go over to the limit $\varepsilon=0$. One obtain an integral over the Euclidean four-momenta $\left(l_{j}\right)_{E}$. The external momenta are pseudo-Euclidean. If $\left[A\left(-k^{2}\right)\right]^{2}=O\left(\frac{1}{k^{2}}\right)$ at $k^{2} \rightarrow-\infty$ then the integrals corresponding to any Feynmann graphs converge. In a similar way one obtains expressions for the amplitude when the functions $A_{\lambda}\left(-k^{2}\right)$ belongs to the type (B).

Thus, the amplitude $T\left(p_{1}, \ldots, p_{n}\right)$ is determined by the convergent integral and depends on the scalar products of the pseudo-Euclidean external momenta $p_{1}, \ldots, p_{n}$. Instead of the pseudo-Euclidean momenta $p_{1}, \ldots, p_{n}$ we introduce $n$ Euclidean momenta $q_{1}, \ldots, q_{n}$.

This can be done since, firstly, any scalar products of $n$ pseudoEuclidean and $n$ Euclidean momenta are defined by the same number of invariant variables and, secondly, the amplitude $T\left(p_{1}, \ldots, p_{n}\right)$ is an analytic function of its invariant variables. Therefore the amplitude $T$ can be considered as dependent on $n$ Euclidean vectors $T\left(q_{1}, \ldots, q_{n}\right)$. The expression obtained is the same as for the true amplitude in the Euclidean domain of space-like or time-like external momenta. Owing to the uniqueness of the analytical continuation the true amplitude $T\left(p_{1}, \ldots, p_{n}\right)$ can be got over the whole region of the change of the invariant variables by means of analytical continuation. 
It should be noted that the suggested procedure is completely equivalent to the usual methods of consideration of the perturbation Feynman graphs. If it is assumed $A_{\lambda}(\square)=1$ then the usual expressions for the scalar amplitudes are obtained.

Thus we get a perturbation series free of the ultraviolet divergences. Now our aim is to check the unitary and causal nature of the series derived.

\section{The Unitarity of the S-matrix}

We show that the constructed $S$-matrix is unitary in each perturbation order on the mass shell, i.e.

$$
\langle\alpha|S S+| \beta\rangle=\langle\alpha \mid \beta\rangle
$$

where $|\alpha\rangle$ and $|\beta\rangle$ are arbitrary physical states. We assume as usual that there exists a set of amplitudes $|n, \mathbf{k}\rangle$ which together with the amplitude of the vacuum state $|0\rangle$ is complete so that for the arbitrary operators $A$ and $B$

$$
\langle\alpha|A B| \beta\rangle=\langle\alpha|A| 0\rangle\langle 0|B| \beta\rangle+\sum_{n} \int d \mathbf{k}\langle\alpha|A| n, \mathbf{k}\rangle\langle n, \mathbf{k}|B| \beta\rangle
$$

and such that the state $|n, \mathbf{k}\rangle$ corresponds to a definite momentum $\mathbf{k}$ and energy $E_{n}(\mathrm{k})>0$. As such a system we choose the following set of states

$$
|n, \mathbf{k}\rangle=a_{\mathbf{k}_{1}}^{+} \ldots a_{\mathbf{k}_{n}}^{+}|0\rangle, \quad\left(\mathbf{k}_{1}+\cdots+\mathbf{k}_{n}=\mathbf{k}\right)
$$

since we are working in perturbation theory. It is assumed that the states $|\alpha\rangle$ and $|\beta\rangle$ in (6.1) may be expanded in the set of states (6.3).

In order to prove the unitarity condition in the form (6.1) it is enough to prove the Cutkosky rule for normal thresholds in the arbitrary Feynmann diagrams. This property was proved by the author [7]. Thus the $S$-matrix constructed is unitary.

Note that the unitarity condition remains valid if a scalar external field $a(x)$ is introduced into the Lagrangian, e.g. in the following way:

$$
\mathscr{L}_{I}(x)=g\left(\Phi^{4}(x)+a(x) \varphi^{2}(x)\right) .
$$

The unitarity in the form (6.1) for the interaction (6.4) is easily proved in each perturbation order if the results of ref. [7] are used.

\section{The Causality Condition for the S-Matrix}

To check the causal nature of our $S$-matrix which is a functional of the operator $\Phi(x)$ we consider the expression

$$
C(x, y)=\frac{\delta}{\delta \Phi(x)}\left(\frac{\delta S}{\delta \Phi(y)} S^{-1}\right) .
$$

In the case of local interaction when the microcausality condition is fulfilled the operator $C(x, y)$ as is known [3] vanishes for $x ₹ y$. Let us find the value of the operator $C(x, y)$ in $(7.1)$ in the case under consideration. 
First of all we show that the operators $\Phi(x)$ in (2.2) satisfy the local commutation relations

$$
\left[\Phi\left(x_{1}\right), \Phi\left(x_{2}\right)\right]_{-}=\left[\varphi\left(x_{1}\right), \varphi\left(x_{2}\right)\right]_{-}=\Delta\left(x_{1}-x_{2}\right)
$$

where $\Delta\left(x_{1}-x_{2}\right)$ is a usual commutator of the scalar field. To this aim we use again improper transition to the limit, i.e. instead of the field $\Phi(x)$ we consider the field $\Phi_{\varepsilon}(x)$ defined by the relation

$$
\Phi_{\varepsilon}(x)=\int d y A_{\lambda}^{\varepsilon}(x-y) \varphi(y)
$$

where $A_{\lambda}^{\varepsilon}(x-y)$ is taken according to (4.18). Then the commutator (7.2) is calculated as follows:

$$
\begin{aligned}
& {\left[\Phi\left(x_{1}\right), \Phi\left(x_{2}\right)\right]_{-}=\lim _{\varepsilon \rightarrow 0}\left[\Phi_{\varepsilon}\left(x_{1}\right), \Phi_{\varepsilon}\left(x_{2}\right)\right]_{-}} \\
& \quad=\lim _{\varepsilon \rightarrow 0} \int d y_{1} \int d y_{2} A_{\lambda}^{\varepsilon}\left(x_{1}-y_{1}\right) A_{\lambda}^{\varepsilon}\left(x_{2}-y_{2}\right)\left[\varphi\left(y_{1}\right), \varphi\left(y_{2}\right)\right]_{-} \\
& \quad=\lim _{\varepsilon \rightarrow 0}\left[A_{\lambda}\left(-m^{2}\right) R^{\varepsilon}\left(m^{2}\right)\right]^{2} \cdot \Delta\left(x_{1}-x_{2}\right)=\Delta\left(x_{1}-x_{2}\right) .
\end{aligned}
$$

The same can be said about the $D^{( \pm)}$-functions of the field $\Phi(x)$

$$
D^{( \pm)}\left(x_{1}-x_{2}\right)=\Delta^{( \pm)}\left(x_{1}-x_{2}\right)
$$

here $\Delta^{( \pm)}$denote the corresponding functions of the scalar field.

We now consider eq. (7.1). Our $S$-matrix is a functional of only the operator of the field $\Phi(x)$. In expanding in a power series in the coupling constant we obtain a series of the form

$$
S=\sum_{n} \frac{1}{n !} \int d^{4} x_{1} \ldots \int d^{4} x_{n} S_{n}\left(x_{1}, \ldots, x_{n}\right): \Phi\left(x_{1}\right) \ldots \Phi\left(x_{n}\right):
$$

where the functions $S_{n}\left(x_{1}, \ldots, x_{n}\right)$ are constructed out of the causal function (2.5) which is the sum of the usual causal function of the scalar field and the distribution $K_{l}\left(x_{i}-x_{j}\right.$ ) (see (5.2)). Insert the series (7.5) into (7.1) and go over again to the $N$-representation. In this case there arise $D^{(-)}$-functions of the operators of the field $\Phi(x)$. According to (7.5) the $D^{(-)}$-function is exactly equal to the $\Delta^{(-)}$-function of the usual scalar field $\varphi(x)$. Therefore if we do not take into account the distributions $K_{l}\left(x_{i}-x_{j}\right)$ we get a usual microcausality condition of the local scalar field, i.e. the operator $C(x, y)$ in (7.1) is zero for $x ₹ y$. But when the distributions $K_{l}\left(x_{i}-x_{j}\right)$ are taken into account in (5.2) the relation (7.1) for $x \approx y$ will be proportional in the second perturbation order of the function $K_{l}(x-y)$ and in the $n$-th perturbation order to a distribution such as

$\int d t_{1} \ldots \int d t_{n-2} K_{l}\left(x-t_{1}\right) K_{l}\left(t_{1}-t_{2}\right) \ldots K_{l}\left(t_{n-2}-y\right)=Q_{(n-1) l}(x-y)$.

Here $Q_{(n-1) l}$ is the distribution of the type investigated with the "elementary" length $l_{1}=(n-1) l$. Hence, in the $n$-th order the operator 
$C(x, y)$ is zero outside the rigious $G$ and $G$ and $G_{(n-1) l}$ where

$$
\begin{gathered}
G: x_{0} \geqq y_{0},(x-y)^{2} \geqq 0, \\
G_{(n-1) l}:-(n-1)^{2} l^{2} \leqq(x-y)^{2} \leqq(n-1)^{2} l^{2} .
\end{gathered}
$$

As was shown in Section 4 the $S$-matrix constructed by us permit the acausal signal to propagate in a certain bounded region which is completely inside $G_{(n-1) l}$ and the shape of which depends on the shape of the impulse of the field investigated or the shape of the wave packets of incident particles.

Thus, strictly speaking, in the complete perturbation series the operator $C(x, y)$ in $(7.1)$ differs from zero everywhere. This means that the S-matrix obtained by us does not obey the causality condition. However, as was said in the first section, in the case of the small coupling constant interaction the violation of the causality at large distances will be rather small.

\section{Conclusion}

It seems to us that the advantage of the scheme developed is the following: firstly, we have succeeded in introducing into the interaction Lagrangian all the ambiguity in the choice of the shape and the value of the "elementary" length; secondly, the amplitudes of the physical processes have no additional singularities in the finite region of change of the invariant momentum variables as compared to the local theory.

In conclusion I expresses my deep gratitude to Professor D. I. BLokhintsev, Academician N. N. Bogolubov and Professor A. A. Logunov as well as Doctor B. M. Medvedev and Doctor I. T. Todorov for discussions.

\section{References}

[1] Wataghin, G.: Z. Physik 88, 92 (1934).

[2] Kirzhniz, D. A.: Uspekhi Fiz. Nauk 90, 129 (1966).

[3] Bogolubov, N. N., and D. V. Shirkov: Introduction to theory of quantized fields. Moscow; Leningrad: Gostekhizdat 1957.

[4] Titchmarsh, E.: Theory of functions. Moscow; Leningrad: Gostekhizdat 1951.

[5] Meiman, N. N.: JETP 47, 1966 (1964).

[6] Cutkosky, R. E.: J. Math. Phys. 1, 429 (1960).

[7] Efimov, G. V.: JNP, 4, 432 (1966). 